\title{
Béatrice Mésini, la coproduction des savoirs : ensemencement et hybridation terrain/théorie
}

\author{
Béatrice Mésini ${ }^{1}$, Nicole Mathieu $^{2}$ \\ 1 Sociopolitologue, CNRS Telemme-MMSH, 13094 Aix-en-Provence cedex 2, France \\ 2 Géographe, UMR Ladyss CNRS, Université de Paris 1, 75005 Paris, France
}

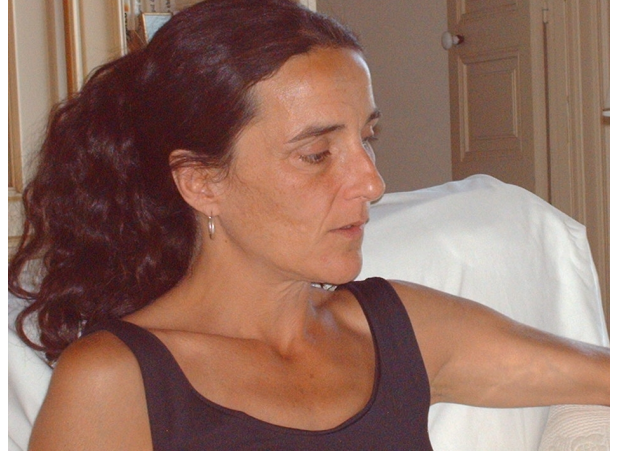

Nicole Mathieu (NSS) : Vous suivez depuis de nombreuses années avec un réel engagement de recherche les mouvements sociaux alternatifs sur les scènes locales, nationales et plus particulièrement mondiales. Pouvezvous me dire, en préambule, quelles sont les raisons de ce mode d'investissement dans la recherche?

Béatrice Mésini : Ce mode d'investissement résulte en premier lieu d'un parti pris éthique puisque dès les années 1990 j'ai choisi de travailler sur les mobilisations et les dynamiques d'action collective, à un moment où les recherches en Sciences sociales se focalisaient sur la nouvelle pauvreté, la galère, la disqualification, la misère et l'exclusion. Il est aussi en partie le fruit d'un hasard puisque recrutée au CNRS en 1996 sur mon thème des « Résistances à l'exclusion dans les villes et les campagnes ", j'ai privilégié les enquêtes en milieu rural, espace qui me semblait progressivement déserté par les chercheurs, surtout par les sociologues. C'est sur la base de cette interconnaissance cognitive avec les mouvements et réseaux paysans de France que j'ai participé au premier Forum social de Millau en 2000, puis à une douzaine d'autres au Brésil, en Europe, en Afrique...

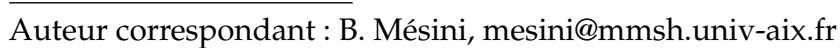

Les forums sociaux constituent un observatoire privilégié pour analyser les alternatives et les luttes rurales ainsi que le processus de convergence et d'internationalisation des revendications contre la marchandisation de la vie, la destruction des peuples, la prédation des territoires et les mésusages de la terre. À titre d'exemple, le Forum mondial de la réforme agraire (FMRA) a réuni en décembre 2004 à Valence, en Espagne, plus de 600 délégués, dont $43 \%$ de femmes, venant de 70 pays du monde, issus de plus de 140 organisations et communautés de base - sociales, agraires, agricoles -, d'organismes de recherche, d'institutions non gouvernementales et gouvernementales ${ }^{1}$. Au centre des débats, la souveraineté alimentaire a été définie comme un nouveau paradigme alternatif reposant sur trois piliers : l'alimentation est un droit de l'homme, les peuples et les États ont le droit de définir leurs propres politiques agricoles, ceux qui produisent les aliments doivent être placés au cœur des politiques publiques. Les participants ont réaffirmé l'intérêt collectif de la réforme agraire, car ce ne sont pas seulement les paysans sans-terre qui réclament l'établissement de mécanismes régulant l'accès aux ressources productives, mais aussi tous ceux dont l'avenir est menacé par la pauvreté, tous ceux qui sont affectés par la destruction des écosystèmes. Recenser ces sources et observer la formation d'alternatives est donc la première raison de mon engagement de chercheur et de

\footnotetext{
${ }^{1}$ Le comité international, promoteur du Forum s'est fédéré sur une base très large de membres dont le Centre espagnol d'études rurales et d'agriculture internationale (CERAI), l'Institut brésilien d'analyse sociale et économique (IBASE), le Centre pour l'étude des Amériques (CENSA), la Fondation pour le progrès de l'Homme (FPH), la Via Campesina, la Confédération nationale brésilienne des travailleurs de l'agriculture (CONTAG), le Mouvement des sans-terre (MST), le réseau international Land Research and Action Network (LRAN), la Plate-forme rurale d'Espagne, Focus on the Global South de Thaïlande...
} 
ma participation au rythme effréné à ces manifestations. Mais la deuxième, associer recherche et construction des alternatives, co-construire les savoirs, qui n'était au départ qu'une hypothèse, s'est trouvée confirmée par cette pratique d'observation des forums sociaux.

Lors de la restitution des avancées du FMRA ${ }^{2}$, j'ai souligné que les délégués participant à l'atelier Recherches extension et technologies pour un modèle paysan du FMRA dénonçaient la privatisation du savoir, à travers les droits de propriété intellectuelle et des brevets, insistant sur l'importance d'un "savoir collectif et communautaire » basé sur l'accès à la formation et à l'information de tous. Pour eux, le secteur privé décide comment et pour qui faire de la recherche, de telle sorte que l'information s'éloigne du paysan. Plusieurs points ont fait l'objet de consensus : quels que soient les modes de recherche et de production de technologies, ils doivent accompagner le processus d'autonomie des peuples paysans et indigènes, marier la culture et les savoirs locaux, et promouvoir la vie en général. Autre point d'accord, les modèles de technologies transférés sont inappropriés car ils provoquent une dépendance croissante, destructrice des rapports sociaux et des ressources locales.

L'un des grands enjeux relevé par ces acteurs est de rapprocher les résultats produits par les centres de recherches publics, les universités et les ONG et les savoirs paysans, souvent sous-estimés voire ignorés de la communauté scientifique. Cela suppose, comme l'argumente Latour ${ }^{3}$, de " changer les tâches assignées d'habitude aux sociologues » car il n'est plus possible de réduire les acteurs au rôle d'informateurs, venant illustrer de façon exemplaire quelque type déjà répertorié, mais il faut leur restituer la capacité de produire leurs propres théories sur le social. C'est ce défi, articuler terrain et théorie par la coconstruction et la coproduction des savoirs entre acteurs et chercheurs, qui fonde depuis quinze ans ma démarche de chercheur.

N.M. : Dans ces forums sociaux les positions et les discours sur les questions agraires sont pour vous centrales à observer et à interpréter, pourquoi ?

Béatrice Mésini : C'est d'abord un apport de connaissances actualisé sur un vaste réseau de solidarité tissé, du local au mondial dans le temps mais aussi dans l'espace, entre mouvements de luttes issus de différents continents. La «multitude » d'associations, syndicats, collectifs et réseaux paysans et indigènes rassemblés lors de forums, contre-sommets et rencontres internationales configure une pluralité de mobilisations en marche pour la défense de la terre, contre la dépossession des droits naturels, culturels, sociaux, coutumiers et positifs. Sont notamment

\footnotetext{
2 Mésini, B., 2005. Le droit de propriété borné par les droits d'usages collectifs, Études foncières, $\mathrm{n}^{\circ} 117$, septembre-octobre 2005.

3 Latour, B., 2006. In Changer de société. Refaire de la Sociologie, Paris, La Découverte, chap. introductif.
}

récurrents les thèmes de préservation de la biodiversité, des cultures (conservation et transmission des pratiques agriculturales mais aussi des cultures indigènes, paysannes, rurales que l'urbanisation croissante menace), des modes de subsistance (contrôle sur les matières premières, souveraineté alimentaire et du droit des peuples à se nourrir) ou encore de souveraineté territoriale, en référence aux minorités indigènes luttant partout dans le monde pour le respect des «droits immémoriaux » sur leurs terres ancestrales.

Cette déclinaison des revendications est à la fois spatiale puisqu'elle s'inscrit dans différents formats de forums sociaux locaux, européens, mondiaux entre 2000 et 2008 mais elle est aussi thématique comme l'indique la tenue des forums sociaux consacrés à la souveraineté alimentaire (La Havane, Cuba, 2001) ainsi qu'à la sécurité alimentaire (Terruel, Espagne, 2002). Ce thème de la souveraineté alimentaire, également au centre du Forum de Sélingué, au Mali, qui a rassemblé 500 participants venus de 80 pays du 23 au 27 février 2007, attestant tout à la fois d'une dynamique des luttes rurales et d'un élargissement de la base paysanne, par la diversité des organisations représentées : pêcheurs traditionnels, peuples autochtones, sans-terre, travailleurs ruraux, paysans migrants, éleveurs nomades, communautés habitant les forêts, femmes, jeunes, consommateurs, mouvements écologistes...

Nul doute que le processus de mondialisation/ globalisation, à l'œuvre en tout point du monde, offre un système d'opportunités politiques ouvert, dans et par lequel convergent les acteurs mobilisés dans les campagnes et milieux ruraux en profondes mutations. Le processus de globalisation agirait ainsi comme un puissant vecteur de «libération cognitive» tel que la définit MacAdam 4 .

N.M. : On sait la méfiance qu'inspirent les travaux des chercheurs quand ils prennent comme champ de recherche des actions sociales - des mouvements sociaux - controversées et fortement marquées par le militantisme. Comment vous y prenez-vous pour étudier ce sujet brûlant qu'est l'altermondialisme et comment menezvous de front engagement et analyse scientifique?

Béatrice Mésini : Dans son livre consacré à la Sociologie des mouvements sociaux publié en 1996, Erik Neveu ${ }^{5}$ soulignait la difficulté des recherches menées sur les mouvements sociaux qui provoquent une «rhétorique de la suspicion ». Ils restent encore largement aujourd'hui diabolisés comme une dangereuse frange extrémiste et radicale de mouvements de lutte, et non comme acteurs quotidiennement en lutte contre l'exploitation, la précarisation et la pauvreté de leurs communautés d'existence.

\footnotetext{
4 MacAdam, D., 1982. In Political Process and the development of Black Insurgency, 1930-1970, The University of Chicago Press, 49.

${ }_{5}$ Neveu, E., 1996. Sociologie des mouvements sociaux, Paris, La Découverte, Repères.
} 
Lire, interpréter et expliciter ces mobilisations et luttes rurales ne consiste pas seulement à replacer les événements aux croisements des faits qui leur donnent une signification première. Dans une perspective positiviste, quelque 4500 sources écrites (en français, anglais et espagnol) ont été collectées in situ. Elles ont été analysées en trois phases distinctes depuis 2000 : en amont des mobilisations, par le croisement des textes d'appels émanant des différents collectifs, associations et réseaux ; durant les rassemblements par la collecte de sources endogènes recueillies sur les stands ${ }^{6}$; en aval enfin, par la création d'une base de données permettant l'analyse croisée des différents niveaux de production de sens.

Le travail d'interprétation prend aussi largement appui sur les retranscriptions et traductions effectuées au retour des rassemblements, des débats, séminaires, ateliers et plénières initiés par les associations du réseau, comme autant de fragments expressifs d'une mémoire narrative ${ }^{7}$. Il s'agit de rendre lisible la diversité des revendications portées par les acteurs des luttes en contrechamp des représentations partielles et partiales, retraduites par les politiques et les médias traditionnels. L'échange avec les acteurs, privilégié comme mode de validation empirique des hypothèses retenues, s'effectue par un retour des transcriptions brutes aux locuteurs, afin de limiter la surinterprétation ou de mésinterprétation des témoignages.

Certains récits sont, intégralement ou partiellement, restitués dans l'analyse pour étudier à la fois la singularité des énoncés, leur variabilité dans le temps, mais aussi dans l'espace, jusqu'à structurer un champ large d'expressivité des conflits et des revendications. Les témoignages restituent aussi une communauté de vue (cosmovision d'une Terre commune), d'expériences et de solidarités, acquises dans la lutte pour l'application de droits garantis ou la définition de nouveaux droits, tant dans les cadres nationaux et internationaux.

N.M. : Est-ce suffisant pour prendre une distance par rapport à la militance de ces acteurs?

Béatrice Mésini : Le fait d'appliquer mon analyse à une pluralité de revendications la décentre de telle sorte qu'elle n'est nullement induite par l'effet leadership de militants phares, mais bel et bien instruite par un constant va-et-vient inductif/déductif des énoncés de chacun(e)s, alimentant pour partie une «mémoire vive » des luttes.

Les limites que je me suis données en retour s'inscrivent, en amont de l'observation, dans une déontologie résolue explicitée auprès des acteurs : pas d' " occupation » de tribunes, libres interventions in situ dans les espaces de débats et d'échanges, pas d'interface médiatique «à

\footnotetext{
${ }^{6}$ Livres, journaux, revues, articles, tracts, pétitions, films.

7 Le retour des transcriptions brutes aux locuteurs est privilégié ici comme mode de validation empirique, afin de limiter les risques de surinterprétation voire de mésinterprétation (traductions) des dires collectés.
}

chaud » mais expressivité des dires en différé. Les sources étayant mes contributions sont par ailleurs systématiquement présentées lors de mes communications et publications dans les sphères scientifique, académique, institutionnelle et associative.

N.M. : Sur quels courants scientifiques et sur quels auteurs vous appuyez-vous pour asseoir et rendre robuste votre démarche?

Béatrice Mésini : Mon travail s'inscrit au croisement de plusieurs champs scientifiques alliant les apports de la géographie sociale, de la sociologie rurale, de la sociologie de l'action et de l'histoire. En ce sens, il s'intègre dans la continuité des travaux féconds du Groupe de sociologie rurale GRS de Nanterre-Paris 10 et de l'équipe rurale du laboratoire de Géographie humaine devenu Strates, deux équipes CNRS réunies en 1996 dans l'UMR Ladyss, qui portaient sur les paysanneries ${ }^{8}$ et les collectivités rurales, les sociétés rurales et classes sociales ${ }^{9}$ mais aussi sur les profondes mutations des espaces ruraux, soumis au double changement de modernisation du secteur agricole et du modèle uniformisant de l'urbanité galopante. Ils s'imprègnent aussi de leurs analyses, menées dans les années 1980, autour des figures d'acteurs militants ${ }^{10}$, des mobilisations paysannes, du développement local qui s'élargissent dans les années 1990 aux problématiques de l'environnement, de la durabilité des territoires ruraux, mais aussi de la pauvreté et de l'exclusion en milieu rural ${ }^{11}$. Enfin, les avancées de terrain renouvellent et redéploient leurs thématiques, saillantes dans les années 2000, d'exode urbain, de conflits d'usages de l'espace rural, de diversité des modes d'habiter ou encore de nouvelles alliances avec la société d'ensemble ${ }^{12}$.

L'analyse emprunte aussi des éléments théoriques à la sociologie des mobilisations et des réseaux. Selon la méthode proposée par Law $(1986)^{13}$, Callon $(2006)^{14}$ et Latour $(2006)^{15}$, la théorie de l'acteur-réseau qui met en scène les relations de pouvoir, les conflits et les luttes devient précieuse pour décrire les interactions entre les «actants » de ces mobilisations. Selon Michel Callon, la

\footnotetext{
8 Mendras, H., 1976. Sociétés paysannes, élément pour une théorie de la paysannerie, Paris, A. Colin.

9 Jollivet, M., 1972. Sociétés rurales et classes sociales, in L'Univers politique des paysans dans la France contemporaine, Tavernier, Y., Gervais, M., Servolin, C. (Eds), Paris, A. Colin.

10 Bourquelot, F., Mathieu, N., 1989. Paroles d'un paysan révolutionnaires : Bernard Lambert, Strates, 4, 129-161.

11 Mathieu, N., 1991. La pauvreté en milieu rural, Alternatives économiques, 12, 12-14.

12 Mésini, B., 2004. Résistances et alternatives rurales à la mondialisation, Études rurales, $\mathrm{n}^{\circ} 169-170,43-60$.

${ }^{13}$ Law, J. (Dir.), 1986. Power, Action and Belief : a new Sociology of Knowledge?, Londres, Routledge, 231-260.

14 Callon, M., 2006. Les réseaux sociaux à l'aune de la théorie de l'acteur-réseau, Sociologies Pratiques, 13, 37-44.

15 Latour, B., 2006. Changer de société. Refaire de la Sociologie, Paris, La Découverte.
} 
notion de réseau comporte plusieurs avantages : elle "permet de se libérer de la distinction entre micro et macrostructure » en circulant du local au global, de «comprendre comment la force ou le pouvoir se construisent par association de faiblesses » et de faire «abstraction du concept de contexte ", les chaînes causales si elles existent étant déployées par les réseaux.

Enfin, ces luttes foncières et agraires ne peuvent se comprendre sans un détour historique et l'analyse de leurs évolutions dans l'espace mondialisé. Dans un livre publié en juin 2008 en collaboration avec Angela Barthes, je fais le lien entre l'internationalisation de ces luttes agraires et foncières et les alternatives déployées en France par les communautés rurales dans les années 1970, les communautés intentionnelles des années 1990 (oasis en tous lieux, écovillages, squats ruraux) ou encore les multiples initiatives associatives visant à la réappropriation des moyens $\mathrm{d}^{\prime}$ existences en milieu rural en France ${ }^{16}$. Ce thème est un sujet mondial qui suppose d'investiguer collectivement les principes qui doivent réguler les droits d'usages de la terre, de l'eau, des ressources et qui réactualise la question des droits collectifs des peuples (sociaux, économiques, culturels, territoriaux). C'est aussi un fait social total qui implique la capacité des sociétés locale, nationale et mondiale à réguler le marché foncier et à garantir l'accès à la terre en vue d'assurer à chacun(e) l'autoréalisation des nécessités vitales : se nourrir, s'abriter.

N.M. : Natures Sciences Sociétés a publié récemment un dossier sur l'engagement du chercheur et s'intéresse au processus même qui met le social en mouvement. Quels sont à votre avis les résultats les plus saillants de vos recherches sur les «mobilisations » paysannes face à la globalisation?

Béatrice Mésini : Cette coalescence des luttes paysannes, agraires, foncières et écologistes, consacre à mes yeux l'irruption de la Terre commune, nouvel acteur collectif doté de droits et sujet de devoirs. Les communautés représentées au centre du Forum mondial de la réforme agraire ont souligné combien tous partageaient une même vision, celle d'une destruction de leurs milieux d'existence.

D'autre part, les témoignages collectés offrent des développements éclairants sur le phénomène de "mémoire manifestante », et plus largement sur l'apprentissage d'une diffusion imitative des répertoires de l'action collective. Tarrow ${ }^{17}$ soulignait en 1996 qu'à une époque

\footnotetext{
16 Mésini, B., Barthes, A. (coll.), 2008. Du local au mondial. Alternatives rurales et luttes paysannes, Université populaire des Alpes-de-Haute-Provence, IUT Digne-les-Bains, ChâteauArnoux.

17 Tarrow, S., 1996. States and opportunities: the political restructuring of social movements, in Comparative perspective on social movements. Political opportunities, mobilizing structures, and cultural framings, McAdam, D., McCarthy, J., Zald, M. N. (Dir.), Cambridge, Cambridge University Press.
}

où les mouvements sociaux se globalisent, ils peuvent de moins en moins facilement être expliqués par les structures d'opportunités politiques propres à un État nation particulier. Si le phénomène de diffusion des modèles d'action collective d'un pays à l'autre n'est pas récent, on assiste aujourd'hui, au-delà de ce diffusionnisme, à une collaboration importante entre mouvements sociaux nationaux dans des campagnes d'envergure, des actions et/ou des marches collectives. De plus en plus de mouvements sont même immédiatement organisés de manière transnationale, procédé stratégique qui ne vise pas uniquement la scène mondiale, mais bien l'espace national : il s'agit d'obtenir un «effet de boomerang », en utilisant la mobilisation transnationale pour pousser les acteurs nationaux à réagir.

Parallèlement à la confluence internationale des mouvements paysans, $j$ 'observe une forte articulation des segments de mobilisation entre les campagnes et les villes, comme l'illustre, en France, la tenue du Forum des luttes du mouvement social, qui a réuni chaque été environ 150 militants des villes et des champs ${ }^{18}$ entre 2000 et 2005, ou encore celle du Réseau de convergence des mouvements sociaux, lancé en juillet 2006 à l'initiative du DAL, Solidaires et de la Confédération Paysanne. Les participants se sont engagés à construire des convergences et des solidarités concrètes, transversales villes/campagnes mais aussi locales, nationales et internationales, ainsi qu'à soutenir et amplifier les mobilisations sociales, en créant de nouveaux espaces de luttes. Dans son livre sur les paysans du monde, Jacques Ollivier ${ }^{19}$ atteste qu'un aspect nouveau des luttes paysannes actuelles concerne le processus de convergence entre les luttes paysannes et rurales et d'autres mouvements sociaux, issus de tous les secteurs de la société. Ce processus témoigne d'une synergie croissante entre des mouvements qui dénoncent des dysfonctionnements de la mondialisation jusqu'à présent peu articulés : les graves atteintes aux écosystèmes terrestres et les crises sociales profondes qui touchent une part croissante de l'humanité.

Un dernier acquis est la mise en évidence de l'indivisibilité des droits humains, sociaux, économiques, agri-culturels, environnementaux. Ces luttes sociales sont protestataires et critiques envers la domination du capitalisme et son lot d'exclusions, mais elles sont aussi largement affirmatives, comme l'indique le répertoire déclaratif des droits fondamentaux des peuples, tant dans les cadres nationaux qu'internationaux. Que l'on soit en Europe, en Afrique, au Brésil, en Inde... la saillance

\footnotetext{
18 La Cisternette (Cévennes) 6-7 juillet 2002, Raventhun (Pasde-Calais), 10-12 juillet 2004, Pernes-les-Fontaines (Vaucluse), 15-17 juillet 2005.

19 Avec les paysans du monde, 2007, édition refondue des $\mathrm{n}^{\circ} 135$ 136, parus en juin-sept. 2006, de la revue Informations et Commentaires - Le développement en question, édité par l'association Pour un nouveau développement.
} 
des revendications en faveur des droits fondamentaux d'existence (à la terre, à la subsistance, au logement, au revenu, à la citoyenneté, à l'éducation, à la santé...) renvoie à un ensemble de prédispositions partagées en termes de privation et de pauvreté "relative ou absolue». En tout état de cause, comme le relèvent Jacques Ion, Spyros Franguiadakis et Pascal Viot (2005), le droit est devenu une ressource centrale pour l'action ${ }^{20}$, et, en retour, la discipline du droit s'approfondit et se renouvelle.

N.M. : En vous plaçant du point de vue d'un chercheur CNRS pouvez-vous conclure sur l'apport de cet «observatoire » aux disciplines proprement dites?

Béatrice Mésini : Pour les disciplines, qu'elles soient de Sciences sociales ou de la nature, la coconstruction des savoirs est un nouvel enjeu qui s'inscrit dans le débat sur le profond changement des rapports qu'entretiennent les sciences et la société. Car la sphère militante, théoriquement formée et bien informée, produit en retour une floraison d'analyses argumentées, les diffuse et les fait circuler en réseau, en lien avec des organismes de recherches officiels ou indépendants. Si les attentes de la société restent fortes à l'égard de la recherche, elles oscillent entre confiance et méfiance à l'égard d'une expertise officielle « en surplomb», produisant ses analyses sans réelle validation auprès des acteurs observés, en particulier dans le domaine des SHS. Sous l'intitulé «Non aux zéros de conduite pour les enfants de 3 ans », la fronde des associations contre le rapport de l'Inserm de septembre 2005 a réuni 30000 signatures contre la «psychiatrisation des manifestations du mal-être social » et conduit à sa condamnation par le Comité national d'éthique. Cette question du conflit d'expertise se pose également avec acuité lors des procès de militants, puisque les travaux de chercheurs invoqués par les accusés et/ou les plaignants, sont parfois contradictoires dans leurs conclusions et/ou mis en concurrence, comme on le voit de façon récurrente sur la question des OGM. À cet effet, je participe au vaste chantier engagé par le CNRS à l'occasion du colloque "Sciences et Société en mutation » qui s'est ouvert à Paris en 2007, car il me semble que, pour dépasser ces controverses stériles, il nous faudrait créer des espaces d'expressivité du dissensus entre chercheurs et/ou acteurs, en amont de la publication des rapports de recherche.

Un autre apport majeur est de décloisonner les frontières des disciplines scientifiques par la pluridisciplinarité et le croisement de problématiques transversales issues des travaux de collègues géographes, sociologues, anthropologues, économistes et juristes. Plusieurs thèmes sont approfondis en contrepoint de ces mobilisations : la re-dissociation des fondements du droit de propriété usus/fructus/abusus, la relativité d'un droit «absolu et sacré » conditionné par ses usages, la disparition orchestrée des terres collectives, la non-brevetabilité du vivant, l'économicisation des biens de subsistance ${ }^{21}$, la reconnaissance des droits ancestraux et immémoriaux, patrimoine commun et/ou communs, l'émergence d'une troisième génération de droits collectifs modulés par les devoirs de chacun, envers la Terre commune ${ }^{22}$. Passer les frontières entre savoirs pour coproduire ceux qui répondent aux nouveaux besoins sociaux, hybrider connaissances « savantes » et «populaires », renouveler la théorie en cultivant le rapport au terrain, tels sont, de mon point de vue, les postulats qui guident ma pratique de recherche.

\footnotetext{
${ }^{20}$ Ion, J., Franguiadakis, S., Viot, P., 2005. in Militer Aujourd'hui, éd. Autrement, 105.

${ }^{21}$ Clément, A., 2006. Les lois économiques doivent-elles s'appliquer aux biens de subsistance?, Cahiers d'économie et sociologie rurales, 79 .

${ }^{22}$ Mésini, B., 2006. Préservation de la biodiversité et souveraineté alimentaire, réactivation des savoirs paysans et émergence des droits de la Terre. France-Europe, Brésil 2000-2005, in Le recours à l'environnement, le retour des paysans, Auclair, Aspe, Baudot (Dir.), Édisud, coll. «Écologie Humaine », 71-90.
} 